\title{
Atypical image of pulmonary alveolar proteinosis — a case report
}

The authors declare no finacial disclosure

\begin{abstract}
Pulmonary alveolar proteinosis is a very rare interstitial lung disease caused by abnormal intra-alveolar surfactant accumulation. Usually, it appears as a "crazy-paving” pattern on high-resolution computed tomography. The image is so typical, that together with the characteristic bronchoalveolar lavage examination with presence of Periodic Acid Schiff positive substance is sufficient for establishing diagnosis, without histological confirmation. We present the case of the young woman with severe dyspnoea suspected of acute hypersensitivity pneumonia. The computed tomography showed numerous intralobular nodules uniformly distributed troughout the lungs. Treatment by corticosteroids had no clinical effect and next computed tomography showed progression. Despite the high risk of complications (patient had a respiratory failure), a surgical lung biopsy was performed and the histopathological diagnosis of pulmonary alveolar proteinosis was made. The whole lung lavage procedure performed twice caused regression of radiological lesions and respiratory failure.
\end{abstract}

Key words: respiratory failure, pulmonary alveolar proteinosis, computed tomography, whole lung lavage

Pneumonol Alergol Pol 2015; 83: 453-456

\section{Introduction}

Pulmonary alveolar proteinosis (PAP) is a rare disease with a prevalence of 3.7-6.2/1 000000. There are three main forms of PAP: hereditary, secondary and autoimmune, but common to all is impaired clearance of surfactant by alveolar macrophages, resulting its retention in the terminal airway and in the alveolar space [1]. A chest computed tomography (CT) commonly showing a „crazy-paving” pattern, is a major tool in the diagnosis of PAP. The image is so typical, that together with the characteristic bronchoalveolar lavage (BAL) examination with presence of Periodic Acid Schiff (PAS) positive substance is sufficient for establishing diagnosis [2]. We present the case of the young woman with severe dyspnoea and radiological image suggesting hypersensitivity pneumonia (HP). The final diagnosis of PAP was established basing on the histological examination of surgical lung biopsy specimens.

\section{Case report}

A 31-year-old caucasian woman admitted to National Research Institute of Tuberculosis and Lung Diseases with a 1 month history of worsening dyspnoea and cough, without fever. She was non-smoker and reported exposure to chemical compounds - last 6 months she worked on the production of car mats (exposure to lead, zinc, solvents) and she had a contact with the pigeons, but it was an accidental exposure. Before her admission she had been treated by empirical antibiotics and bronchodilators without improvement.

On admission she was in severe general condition. Respiratory rate was 28 breaths per min, heart rate 120 beats per min, and oxygen

Address for correspondence: Justyna Fijołek, The Third Department of Pneumonology, National Research Institute of Tuberculosis and Lung Diseases, Plocka 26, 01-138 Warsaw, Poland, e-mail: jiijolek@op.pl

DOI: 10.5603/PiAP.2015.0074

Received: 02.07.2015

Copyright (C) 2015 PTChP

ISSN 0867-7077 
saturation was $68 \%$ on room air. Chest examination showed good air entry with no abnormal sounds. Her full blood count, C-reactive protein, antinuclear antibodies and complement levels were normal, but she had a raised lactate dehydrogenase (LDH) concentration of $1123 \mathrm{U} / \mathrm{L}$ (normal 200-384 U/L). Pulmonary function tests showed reduced diffusing capacity of the lung for carbon monoxide (DLCO) to a severe degree $(35 \% \mathrm{~N})$. Chest radiograph showed bilateral non-specific disseminated lesions and high resolution computed tomography (HRCT) appeared numerous intralobular nodules uniformly distributed throughout the lungs (Figure 1). Based on the HRCT image together with the exposure to the pigeons' antigens in medical history, HP was strongly suspected. Patient received $30 \mathrm{mg}$ of prednisone without clinical improvement and respiratory failure remained $\left(\mathrm{PaO}_{2}=32-40 \mathrm{~mm} \mathrm{Hg}\right)$. She required oxygen supplementation $3-5 \mathrm{~L} / \mathrm{min}$, despite increased dose of corticosteroids (CS) to $60 \mathrm{mg}$ daily. Control HRCT showed a progression of the intralobular opacities with the appearance of the discrete „septal thickening”. Bronchoscopy with transbronchial lung biopsy (TBLB) was performed, but histological examination was not conclusive. All cultures were negative. Patient underwent bronchoalveolar lavage, but the procedure had to be stopped, because of severe dyspnoea with decrease of the oxygen saturation. After 3 weeks of corticotherapy without marked improvement, surgical lung biopsy was performed to determine the diagnosis. The histopathological

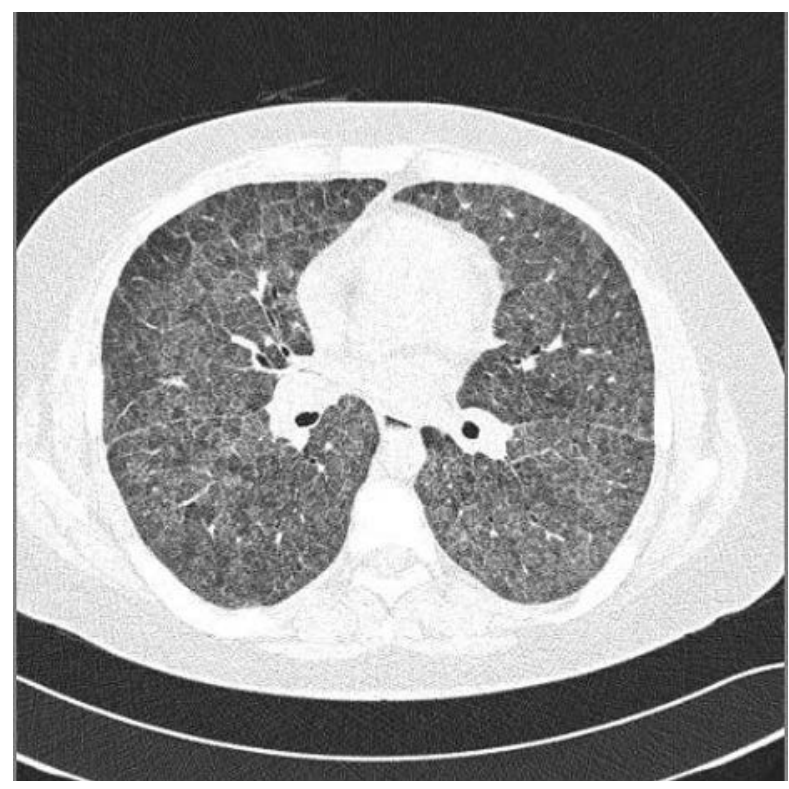

Figure 1. High-resolution computed tomography scan showing atypical image of PAP - numerous diffuse intralobular nodules

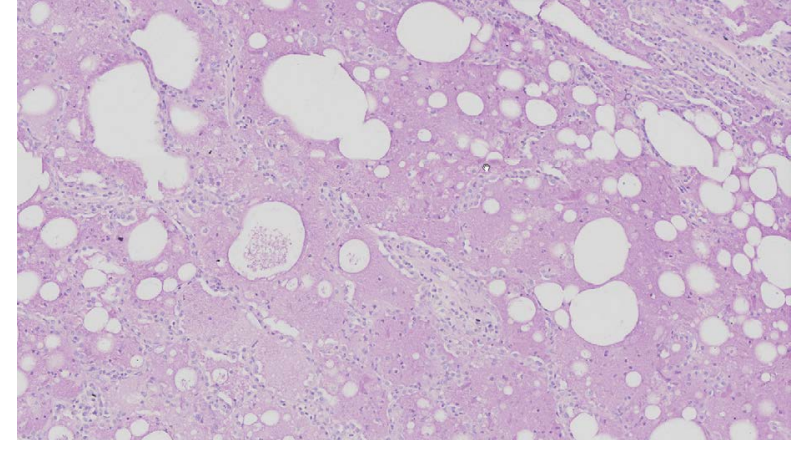

Figure 2. Surgical biopsy photomicrograph - alveoli filled with PAS-positive staining substance (original $\times 400$ )

examination revealed alveoli and terminal bronchioles filled with an eosinophilic proteinaceous material that stained pink with PAS - the diagnosis was PAP (Figure 2). Anti-granulocyte macrophage - colony stimulating factor (anti-GM-CSF) antibody concentrations - the gold standard for diagnosis of autoimmune PAP - is not available in Poland. The CS were gradually eliminated and stopped and whole lung lavage (WLL) with $10 \mathrm{~L}$ of $0.9 \%$ saline was performed (in the operating room under general anaesthesia). Directly after one procedure, the clinical improvement was observed. At last follow-up after 6 weeks, the patient was asymptomatic and chest radiograph showed regression of the disseminated lesions (Figure 3A, B).

\section{Discussion}

PAP is a rare syndrome characterized by the accumulation of surfactant - like substance in pulmonary alveoli resulting in varying degrees of respiratory insufficiency and myeloid cell dysfunction resulting in increased risk of infection [1]. Autoimmune PAP occurs, when anti GM-CSF antibodies block activation of alveolar macrophages. It is the most common type of PAP and accounts for $90 \%$ cases. Congenital PAP is caused by mutations in surfactant proteins genes B or C, ABCA3 and a chain of the GM-CSF receptor. Secondary PAP is an acquired defect of malignancies, inhalation of inorganic agents or opportunistic infections [3]. Our patient had exposure to chemical compound, like lead, zinc and solvents during last 6 months, which may suggest the secondary type of the disease, but recent studies showed, that a history of chemical substances inhalation may be strongly associated also with the autoimmune form of PAP [4]. A Differentiating factor could be examination of the 


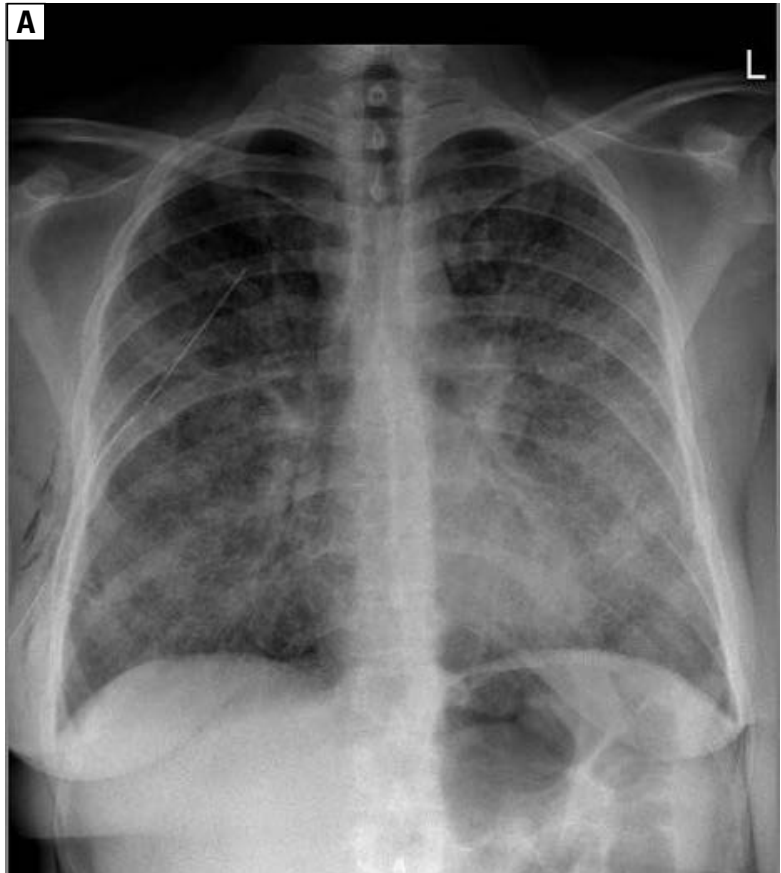

Figure 3A. Chest radiograph before whole lung lavage - visible disseminated lesions in the lungs

anti-GM-CSF antibody concentration in patient's serum, but in Poland it is not available. On the other hand, autoantibodies are detected also in secondary PAP, but are much less common [5]. IgG anti-GM-CSF have been described in a patient with PAP secondary to exposure to indium [6].

The most often symptoms of PAP are dyspnoea and cough (39-79\%), which are common, but non-specific symptoms seen by medical practitioners [3]. An important examination for the diagnosis of PAP is HRCT, which shows lesions in the form of ground-glass opacities with visible polygonal structures - the so-called „crazy paving” pattern. The areas of filled alveoli are usually separated from the normal lung parenchyma making a geographical pattern (Figure 4) [7]. Vrielynck et al. evaluated the accuracy of chest CT in the differential diagnosis of chronic infiltrative lung disease in children. Among a cohort of 59 patients with nine disorders, PAP was the most frequently properly diagnosed $(72 \%)$, showing that the HRCT appearance of PAP is suggestive of the disease [8]. Current guidelines imply, that characteristic image of HRCT together with a typical results of the examination of BAL are sufficient criteria for establishing diagnosis, without histological confirmation [2, 7]. Unfortunately, in our patient we have not received material from BAL, because of severe respiratory failure. On the other hand, HRCT showed multiple intralobular nodules uncharacteristic for PAP and in this

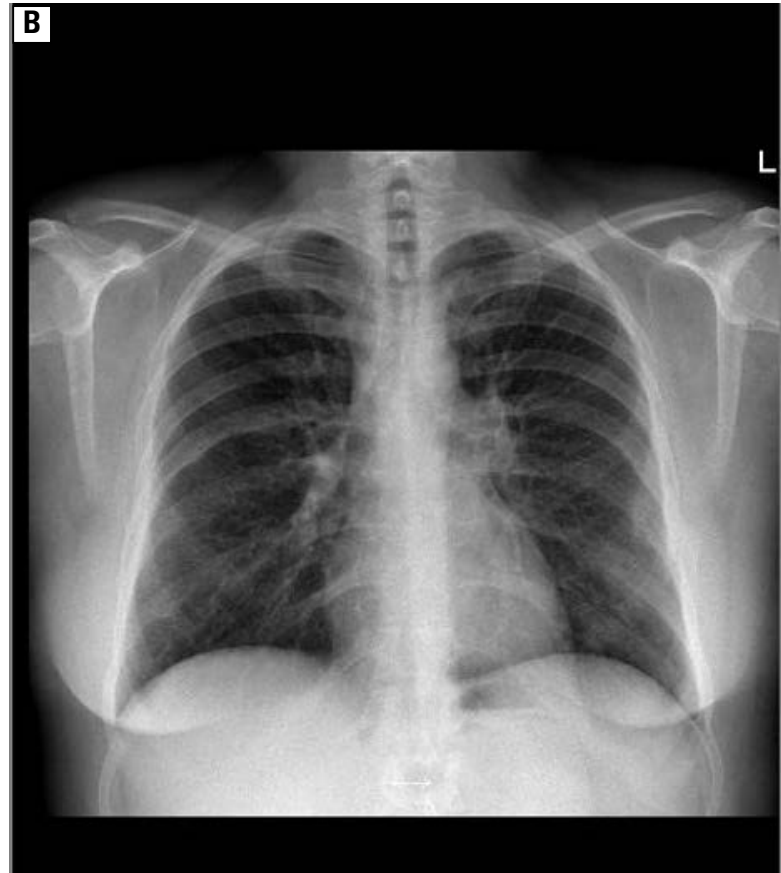

Figure 3B. Chest radiograph after whole lung lavage - complete regression of lesions

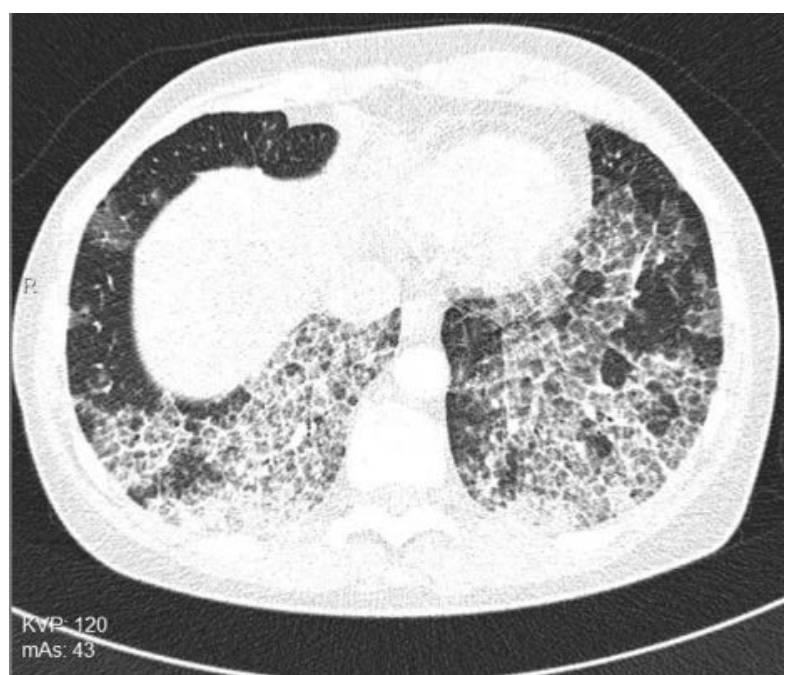

Figure 4. High-resolution computed tomography scan showing typical image of PAP: ground-glass opacities with visible polygonal structures - the so-called "crazy paving" pattern (from own collection of the Third Department of Pneumonology, National Research Institute of Tuberculosis and Lung Diseases)

situation typical BAL examination could not be sufficient for diagnosis of PAP. Clinical course of disease was against of HP diagnosis and surgical lung biopsy was necessary. Perhaps, the decision to proceed surgical biopsy could be taken at the time the lack of therapeutic effect of $30 \mathrm{mg}$ of prednisone, but patient was in a serious condition with respiratory failure and we wanted to take the 
opportunity of optimal treatment, before deciding on the invasive procedure. Our case shows, that HRCT image of PAP can be atypical and uncharacteristic, which may hinder correct diagnosis. Intralobular nodules observed in our patient conform an unusual and occasional manifestation of PAP. In the Holbert's et al study investigating the HRCT features of PAP in a group of 27 patients, intralobular lesions were found only in two cases (7.4\%) [9], while Berteloot et al found intralobular nodules only in one case of PAP (4.2\%) [10]. In the material of Fijolek et al., among the 17 patients with PAP, in 4 cases another interstitial diseases were considered, but PAP was also taken into account [11]. Although the nodular appearance of PAP on HRCT could not be clearly explained, the appearance of such pattern may suggest very early PAP in patients.

There are no firm criteria for initiation of therapy of PAP, but according to the majority of authors, the treatment is necessary in case of hypoxaemia $\left(\mathrm{PaO}_{2}<65 \mathrm{~mm} \mathrm{Hg}\right.$ or $\left.<60 \mathrm{~mm} \mathrm{Hg}\right)$ or saturation below $90 \%$, the growth of alveolocapillary gradient of oxygen partial pressure above $40 \mathrm{~mm} \mathrm{Hg}$, pulmonary shunt exceeding $10-12 \%$ and/or dyspnoea limiting daily activities of the patient $[12,13]$. Still the most effective and safe method of treatment is WLL, which removes surfactant-like material from the alveoli $[7,11$, 14]. The improvement starts within days and is sustainable in many. Usually, oxygenation improves first, chest imaging shows varying degrees of resolution, but complete resolution of opacities is uncommon. Our patient met the eligibility criteria for treatment and improvement was quickly observed - few days after WLL blood gases normalized, and follow-up HRCT image performed 6 weeks later showed almost complete regression of the disease.

In conclusion, radiological image of PAP at HRCT is usually typical and it is decisive for diagnosis, but sometimes HRCT is not characteristic and may suggest other disease. Bilateral diffuse intralobular nodules can be caused by infection, panbronchiolitis, vasculitis and vascular metastases, respiratory bronchiolitis-interstitial lung disease, HP and pneumoconiosis [15]. Our case shows, that in case of disseminated intralobular nodules in CT, PAP - although exteremely rare - should be taken into consideration in differential diagnosis. Our conclusion is, that in cases of undiagnosed diffuse pulmonary disease, espe- cially in young patients the surgical lung biopsy should be performed. The precise diagnosis is necessary to implement appropriate treatment, without exposing the patient to the inadequate therapy and its possible side effects.

\section{Acknowledgements}

The authors thank the patient for her participation.

\section{Conflict of interest}

The authors declare no conflict of interest.

\section{References:}

1. Borie R, Daniel C, Debray M-P et al. Pulmonary alveolar proteinosis. Eur Respir J 2011; 20: 98-107. doi: 10.1183/09059180.00001311.

2. Wells AU, Hirani N. Interstitial lung disease guidelines: the British Thoracic Society in collaboration with the Thoracic Society of Australia and New Zealand and the Irish Thoracic Society. Thorax 2008 (suppl. V): 1-58. doi: 10.1136/ thx.2008.101691.

3. Seymour JF, Presneill JJ. Pulmonary alveolar proteinosis: progress in the first 44 years. Am J Respir Crit Care Med 2002; 166: 215-235.

4. Bonella F, Bauer PC, Griese M et al. Pulmonary alveolar proteinosis: new insights from a single-center cohort of 70 patients. Respir Med 2011; 105: 1908-1916. doi: 10.1016/j. rmed.2011.08.018.

5. Ben-Dov I, Segel MJ. Autoimmune pulmonary alveolar proteinosis: clinical course and diagnostic criteria. Autoimm Rev 2014; 13: 513-517. doi: 10.1016/j.autrev.2014.01.046.

6. Cummings KJ, Donat WE, Ettensohn DB et al. Pulmonary alveolar proteinosis in workers a tan indium processing facility Am J Respir Crit Care Med 2010; 181: 458-464. doi: 10.1164/ rccm.200907-1022CR.

7. Huizar I, Kavuru MS. Alveolar proteinosis syndrome: pathogenesis, diagnosis and management. Curr Opin Pulm Med 2009; 15: 491-498. doi: 10.1097/MCP.0b013e32832ea51c.

8. Vrielynck S, Mamou-Mani T, Emond S et al. Diagnostic value of high-resolution CT in the evaluation of chronic infiltrative lung disease in children. AJR 2008; 191: 914-920. doi: 10.2214/AJR.07.2710

9. Holbert JM, Costello P, Li W et al. CT features of pulmonary alveolar proteinosis. AJR 2001; 176: 1287-1294.

10. Berteloot L, Taam RA, Emond-Gonsard S et al. Primary pulmonary alveolar proteinosis: computed tomography features at diagnosis. Pediatr Radiol 2014; 44: 795-802. doi: 10.1007/ s00247-014-2888-1.

11. Fijolek J, Wiatr E, Radzikowska E et al. Pulmonary alveolar proteinosis during 30-years observation. Diagnosis and treatment. Pneumonol Alergol Pol 2014; 82: 206-217. doi: 10.5603/ PiAP.2014.0028.

12. Michaud G, Reddy Ch, Ernst A. Whole-lung lavage for pulmonary alveolar proteinosis. Chest 2009; 136: 1678-1681. doi: 10.1378/chest.09-2295.

13. Khan A, Agarwal R. Pulmonary alveolar proteinosis. Respir Care 2011; 56: 1016-1028.

14. Wiatr E, Prokopowicz J, Grudny J et al. Whole-lung lavage in alveolar proteinosis. Pneumonol Alergol Pol 2003; 71: 74-85.

15. Lee KS, Kim TS, Han J et al. Diffuse micronodular lung disease: HRCT and pathologic findings. J Comput Assist Tomogr 1999; 23: 99-106. 